\title{
El aprendizaje lúdico como estrategia didáctica: El territorio en Juego
}

Asenet Sosa Espinosa ${ }^{a}$, Eric Gielen', José Sergio Palencia Jiménez ${ }^{\text {, }}$ Yaiza Pérez Alonso $^{\text {d, María Salomé Moreno Navarro }}{ }^{\mathrm{e}}$, Rafael Ramón Temes Córdovez , Jose Luis Miralles Garciag, Maria Mercedes Trenor Galindo ${ }^{\text {h }}$

a Departamento de Urbanismo, assoes@urv.upv.es; ${ }^{\mathrm{b}}$ Departamento de Urbanismo, egielen@urb.upv.es; ${ }^{\mathrm{c}}$ Departamento de Urbanismo, ipalenci@urb.upv.es; ${ }^{\mathrm{d}}$ Departamento de Urbanismo, yaipeal@urb.upv.es; eㅡepartamento de Urbanismo, smoreno@upvnet.upv.es; fDepartamento de Urbanismo, rtemesc@urb.upv.es; ${ }^{\mathrm{g}}$ Departamento de Urbanismo, jlmirall@urb.upv.es; ${ }^{\mathrm{h}}$ Departamento de Urbanismo,. mtrenor@urb.upv.es.

\begin{abstract}
The game learning as a didactic strategy in matters of Urban and Regional Planning is the challenge of the Project of Innovation and Educational Improvement (PIME) "The territory in play". The project intend to build a teaching-learning environment that encourages students to create their own knowledge and experience, through the design of a board game. The proposal is designed to alleviate the weaknesses and threats detected, strengthen the strengths and take advantage of the possibilities of the didactic play resource, to improve learning, apply knowledge, reflect on the territory and motivate students.

The results of the first phase of the PIME are presented here, in which using a methodology based on implicative and reflexive techniques, three commercialized games are tested. The games tested propose extreme situatins: absolutely cooperative, absolutely competitive, with collaboration being a commercial instrument and sustainability a principle that is little contemplated or nullified. However, each provide elements and that, properly combined into the very model of game playing techniques interesting, may be useful for improving learning and therefore the results of students of Urban and Regional Planning, ultimate end of PIME that is presented here.
\end{abstract}

Keywords: game learning, active methodology, cooperation-opposition game, spatial planning

\section{Resumen}

El aprendizaje lúdico como estrategia didáctica en materias de Ordenación del Territorio y Urbanismo es el reto del Proyecto de Innovación y Mejora Educativa (PIME) "El territorio en juego". Se pretende construir un entorno de enseñanza-aprendizaje que estimule 
al alumnado a crear su propio conocimiento y experiencia, a través del diseño de un juego de mesa. La propuesta está diseñada para paliar las debilidades y amenazas detectadas, potenciar las fortalezas y aprovechar las posibilidades del recurso lúdico didáctico, para mejorar el aprendizaje, aplicar conocimientos, reflexionar sobre el territorio y motivar al alumnado.

Se presentan aquí los resultados de la primera fase del PIME, en la cual utilizando una metodología basada en técnicas implicativas y reflexivas, se testean tres juegos comercializados. Los juegos testeados nos sitúan ante situaciones que se posicionan en los extremos: absolutamente cooperativo-absolutamente competitivo, siendo la colaboración un instrumento comercial y la sostenibilidad un principio escasamente contemplado o anulado. Sin embargo, cada uno de ellos proporcionan elementos y técnicas de juego interesantes que, adecuadamente combinados en el modelo propio de juego, pueden ser útiles para mejorar el aprendizaje y por tanto los resultados de los alumnos de Urbanismo y Ordenación del Territorio, fin ultimo del PIME que aquí se presenta.

Palabras clave: aprendizaje lúdico, metodología activa, juego de cooperación-oposición, ordenación del territorio.

\section{Introducción}

El presente trabajo se aborda a partir de los fundamentos y claves en los que inscribe el Proyecto de Innovación y Mejora Educativa (PIME) denominado "El aprendizaje lúdico como estrategia didáctica: el territorio en juego", perteneciente a la convocatoria de Proyectos de Innovación y Mejora Educativa del Vicerrectorado de Estudios, Calidad y Acreditación de la Universitat Politècnica de València, y cofinanciado por la Escuela Superior de Ingenieros de Caminos, Canales y Puertos, correspondiente a la convocatoria de 2017 y que llegará a su fin en 2019.

El proyecto nace de la experiencia docente en aquellas asignaturas relacionadas con el área de conocimiento del Urbanismo y de la Ordenación del Territorio que actualmente se imparten en las escuelas de Ingeniería de Caminos, Canales y Puertos, de Arquitectura y de Ingeniería Geodésica, Cartográfica y Topográfica de la Universitat Politècnica de València, a lo que se une el conocimiento del profesorado implicado en el proyecto en juegos de mesa relacionados con las competencias necesarias para abordar una disciplina tan compleja y con un alto nivel de abstracción, como es el Urbanismo y la Ordenación del territorio. 
Desde hace varios años, en la universidad se está produciendo un descenso en el número de alumnos matriculados en las carreras de ingeniería que se encuentran directamente relacionadas con la obra civil y la construcción en general. Este hecho ha supuesto un descenso en la exigencia de acceso a este tipo de titulaciones, tradicionalmente elegidas por alumnos con un alto expediente académico. De este modo, se ha producido una modificación en el perfil del alumnado de nuevo ingreso de la universidad, que acceden a la misma con una nota de corte de $5^{1}$, lo que, salvo excepciones, supone que el alumnado medio actual que se matricula en estas ingenierías accede con un nivel académico mediobajo. Además, su primera opción no era el título en el que se matricula y por lo tanto, accede con una menor motivación que el matriculado años anteriores.

Por otro lado, las asignaturas de Ordenación del Territorio y Urbanismo son complejas. Se trata de una disciplina con un enfoque transversal y global, lo que supone la incorporación necesaria de un pensamiento complejo de alto nivel y de abstracción lo que dificulta el aprendizaje profundo en el alumno. Esto último constituye un aspecto de especial importancia a tener en cuenta en la propuesta educativa de esta disciplina. La problemática se acentúa aún más en los primeros cursos, especialmente en primero y segundo curso de grado, donde el alumnado no dispone del bagaje y la madurez suficiente para abordar con éxito estas temáticas. Todo ello supone una dificultad añadida al aprendizaje del estudiante, así como una escasa motivación, que se pone de manifiesto en las asignaturas troncales de los primeros cursos (71 estudiantes en la asignatura de Transporte y Territorio del Grado de Ingeniería Civil, 46 estudiantes en Urbanismo y Territorio del Grado en Ingeniería de Obras Públicas y 96 estudiantes en Urbanismo y Ordenacion del Territorio en el Master de Caminos, Canales y Puertos). Como consecuencia de todo lo anterior, nos encontramos, en los cursos siguientes, con una bajo índice de matriculación en las asignaturas optativas adscritas a esta área de conocimiento: es el caso de la optatividad en tercero y cuarto tanto en el Grado de Ingeniería Civil como en el Grado en Ingeniería de Obras Públicas, así como en el Master de Caminos, Canales y Puertos.

De la observación de esta realidad en los últimos años, nace la propuesta del Proyecto de Innovación y Mejora Educativa (PIME), cuyo objetivo es el de crear un juego de mesa como recurso lúdico-educativo para mejorar el proceso de aprendizaje en las asignaturas de Urbanismo y Ordenación del Territorio, bajo los principios de la cooperación y la competición, en el que se pone de manifiesto el conflicto y el consenso sobre decisiones territoriales. Todo esto se canaliza, mediante la asunción de roles que representan los distintos agentes sociales que, actúan dentro de un marco definido en las propias reglas del juego, que pretenden reproducir los criterios básicos de lo que supone una ocupación racional y sostenible del suelo. De este modo, para ganar, los jugadores compiten entre sí para cumplir sus objetivos, pero para ello es necesario que la "sociedad" creada alcance

\footnotetext{
${ }^{1}$ En las 2 titulaciones oficiales de grado de la Escuela de Caminos Canales y Puertos involucradas en el PIME, Grado de Ingeniería Civil y Grado en Ingeniería de Obras Públicas, según datos de Informe 2015-2016 de la Dirección General de Universidad y Estudios Superiores de la GVA
} 
ciertos niveles de "calidad y bienestar social", siendo necesario para ello que se trabajen valores transversales como la colaboración y la sostenibilidad.

El PIME se estructura en distintas fases entre los que se encuentra, en primer lugar, testear la idoneidad del uso de juegos como recurso didáctico para la enseñanza de la ordenación del territorio, como elemento motivador del aprendizaje del alumnado. En este trabajo se presentan los resultados obtenidos en la primera fase realizada por el equipo docente de 10 profesores del Departamento de Urbanismo de la Universitat Politècnica de València que compone el PIME: El aprendizaje lúdico como estrategia didáctica: el territorio en juego, el testeo de diferentes juegos comerciales.

\section{Objetivos}

El objetivo general de esta comunicación es presentar los resultados del testeo de tres juegos comerciales utilizados como recurso didáctico para la ordenación del territorio por el equipo docente que desarrolla el PIME, y que se inscribe en la primera fase de dicho proyecto. A partir de dicho objetivo general tenemos otros objetivos específicos:

1. Describir la metodología empleada en el testeo de los juegos.

2. Extraer el aprendizaje que para el urbanismo y la ordenación del territorio, nos ofrecen diferentes juegos comerciales

\section{Fundamentos y desarrollo de la innovación}

El juego didáctico es una estrategia de enseñanza y aprendizaje que se puede usar en cualquier etapa educativa. La actividad lúdica es atractiva, motivadora y capta la atención (Chacón, 2008). Además, la relación entre juego y aprendizaje es natural pues desde la infancia el juego es parte del aprendizaje. Su utilización es relativamente común en la enseñanza de las matemáticas en edades tempranas. Sin embargo, su presencia es muy limitada en el ámbito universitario (Gaete-Quezada, 2010). Esto se debe a dos motivos principales: en primer lugar, el desconocimiento de los docentes en cuanto a sus ventajas (Chacón, 2008); y en segundo lugar, por una docencia universitaria muy a menudo anclada en paradigmas tradicionales, donde el profesor tiene un rol central y el alumno y la alumna un rol pasivo (Gaete-Quezada, 2010). El juego en aula proporciona una herramienta que facilita la comprensión de los contenidos teóricos, pues la complejidad para comprender la teoría está vinculada con los distintos niveles de abstracción: lo intangible es más complejo de comprender que lo tangible. El recurso lúdico-didáctico que aquí se propone, pretende precisamente investigar cómo llevar la complejidad territorial al aula de forma tangible (Sosa y Peréz, 2014:60).

Entre los diferentes juegos se pueden establecer "tipos" relacionados con el proceso educativo (Ripoll, 2006:17-18):

- Juegos individuales: el jugador no tiene oponentes, juega superando hitos alcanzados en partidas anteriores.

(cc) EY-NC-ND 2018, Universitat Politècnica de València

Congreso IN-RED (2018) 
- Juegos de oposición: el jugador no tiene compañeros pero sí oponentes y debe jugar para superarlos.

- Juegos de cooperación: no existen oponentes, solo compañeros. Entre todos se tiene que superar un reto, buscando una estrategia común.

- Juegos de cooperación-oposición: existen compañeros y oponentes. Cada uno de los grupos juega a superar al resto buscando estrategias comunes que resulten óptimas.

El juego constituye una gran oportunidad para la mejora del aprendizaje y eso resulta particularmente interesante en lo que se refiere a la adquisición de competencias en Urbanismo y Ordenación del Territorio. Con el uso de este tipo de estrategia lúdica, se pretende poner en situación al alumnado, construyendo un entorno que estimule al estudiante a crear su propio conocimiento y experiencia (Chacón, 2008), pues permite trabajar todas las fases del proyecto; desde el establecimiento de objetivos, el diagnóstico y el establecimiento de una propuesta, todo ello, "pasando por la divergencia o generación de ideas, hasta la convergencia en soluciones prácticas. Permiten además, romper las barreras y mejorar la comunicación en el grupo y generar nuevas ideas, visiones y estrategias" (Gonzalez, 2014:4). Con el juego se aprende a hacer, lo cual permite al alumnado la adquisición de competencias para su calificación profesional y enfrentarse a situaciones diversas y trabajar en equipo, dentro de los marcos sociales y laborales donde desarrolla su vida (Delors, 1996: 34).

De este modo, el juego se convierte en una potente herramienta para abordar la enseñanza-aprendizaje dentro de disciplinas complejas y con alto nivel de abstracción como son el Urbanismo y la Ordenación del Territorio. Esta disciplina posee un enfoque multicriterio centrado en la solución de problemas, en base a un trabajo interdisciplinario, donde intervienen múltiples agentes con intereses diferenciados y, por lo tanto, propuestas de actuación también diferenciadas. Todo este proceso culmina en la toma de decisiones después de un complejo trabajo de integración y aprendizaje permanente.

Con enfoques similares, existen experiencias en técnicas de simulación y juegos para la educación ambiental ya antiguas como el "Modelo CLUG (Community Land Use Game)", diseñado por Allan Felt (1972). Se trata de un juego de tablero cuyas reglas son representativas de los factores que intervienen en la forma del crecimiento urbano, reproduciendo las interacciones en la economía y desarrollo del suelo (Taylor, 1983).

En el ámbito educativo, "Flood Resilience Game” es un juego desarrollado por "Zurich Flood Resilience Alliance y el Institute International of Applied Systems Analysis", que se ha usado en Perú para concienciar y educar a los ciudadanos que viven en zonas inundables. Existen otros ejemplos en tablero como "The Game of Urban Renewal". Sobre esta temática también existen algunas app educativas como "Move a lot" desarrollado para Android por la oficina catastral holandesa (Kadaster).

También, se han desarrollado comercialmente numerosos juegos de estrategia, que serán también objeto de análisis en este trabajo. 


\section{Metodología}

El Proyecto de Innovación y Mejora Educativa mencionado presenta una articulación metodológica por complementación encadenada, esto es, la combinación de la metodología cuantitativa y cualitativa en diferentes fases consecutivas.

Para la fase primera, en la que se inscribe el testeo de juegos, se utilizó metodología cualitativa, concretamente una técnica implicativa y reflexiva denominada "matriz de escenarios presentes y futuros” (Montañés, 2009:121). Este tipo de herramienta permite la construcción colectiva de una matriz diagnóstico que da visibilidad a los aspectos, tanto positivos como negativos, presentes en el juego que se analiza, así como aquellos que pueden mejorar o pertubar la aplicación docente del juego en materias que tienen que ver con el Urbanismo y la Ordenación del Territorio. Así se definen cuatro cuadrantes, dos positivos y dos negativos enfrentados: "lo que hay y me gusta, lo que hay y no me gusta, lo que no hay y me gustaría que hubiera y lo que no hay y no me gustaría que hubiera”.

Dicha metodología se aplicó, concretamente, a tres juegos que están en el mercado y que pasamos a tipificar brevemente.

\section{1.-Juegos seleccionados.}

La selección de los juegos de tablero objeto de análisis se realizó bajo principios que nos permitiesen abarcar diferentes modalidades presentes: de estrategia colectiva y/o individual, colaborativo y/o competitivo, cubriendo de esta forma la tipificación realizada por Ripoll (2006) y recogida en líneas anteriores.

Los juegos de mesa seleccionados y sus características básicas son las siguientes:

- Honshú (Fig. 1): es un juego de losetas (en este caso cartas), diseñado por Kalle Malmioja y editado por “Games for Gamers", que se desarrolla en varias rondas de juego. En cada una de ellas pujaremos por obtener una carta de mapa que una vez adquirida colocaremos en nuestro mapa inicial. El objetivo es ir ampliando nuestro territorio en los diferentes tipos de terreno existentes para obtener puntos de victoria al final de la partida, según la carta de resumen de puntuación multicriterio que cada uno tiene.

Se trata de un un juego individualista y de oposición. Cada participante juega solo y compite con los otros jugadores en la construcción y ordenación de un territorio sin una base previa, a través de un sistema de cartas. Su carácter es estructural y estratégico.

(cc) EY-NC-ND 2018, Universitat Politècnica de València

Congreso IN-RED (2018) 


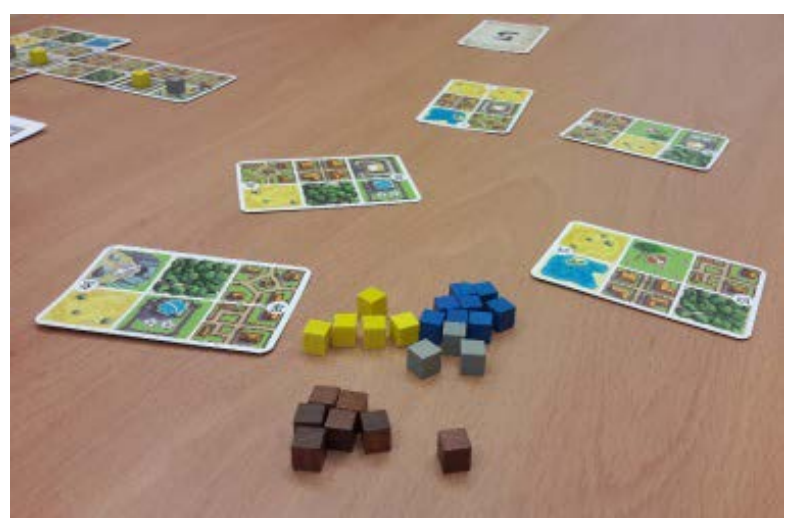

Fig. 1 Juego Honsú

- Catán en sus modalidades básica y extensión recurso petróleo (Fig. 2): es un clásico juego de estrategia alemán inventado por Klaus Teuber y editado en España por Devir Iberia donde, en su versión básica, los jugadores compiten para ver quien consigue colonizar una isla de una manera más ordenada y eficiente, recolectando las materias primas necesarias, así como negociando con los demás jugadores para conseguir esos recursos que necesitan para construir caminos, aldeas, ciudades, puertos, etc. Sobre este mismo juego, se ha desarrollado una versión no comercial y de uso libre llamada "Oilsprings". Se trata de un escenario ecológico de la versión de base donde el jugador tiene que lidiar con el agotamiento de los recursos cambio climático y otros desafíos ambientales que se producen como consecuencia de un consumo excesivo de recursos ${ }^{2}$.

Catán es un juego individualista y de oposición. Cada participante juega solo y compite con los otros jugadores en la dominación de territorio. Permite negociar con otros jugadores para la adquisión de recursos y evitar riesgo ecológico. Posee tablero que delimita el territorio. Su carácter es estructural y estratégico.

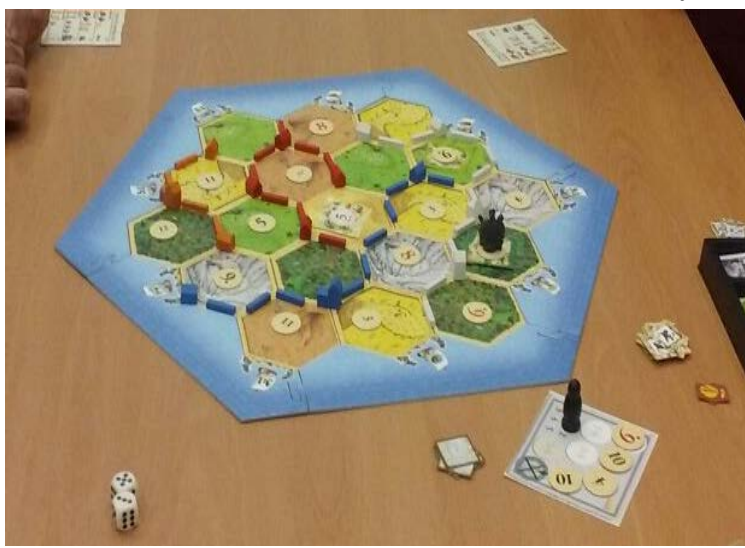

Fig. 2. Juego Catán

\footnotetext{
${ }^{2}$ http://www.oilsprings.catan.com
} 
- $\quad$ La Isla Prohibida (Fig. 3): es un juego colaborativo, diseñado por Matt Leacock y editado por Devir Iberia en España, en el que los jugadores deben evacuar una mítica isla misteriosa y para ello han de conseguir unos tesoros, necesarios para la evacuación de las personas, antes de que las aguas la inunden por completo y el territorio quede sumergido.

Se trata de un juego de cooperación, ganan todos o pierden todos, en la superación del reto (riesgo) que el propio juego establece. Posee un tablero que delimita el territorio. Su carácter es estratégico y requiere la toma de decisiones consensuadas por todos los participantes.

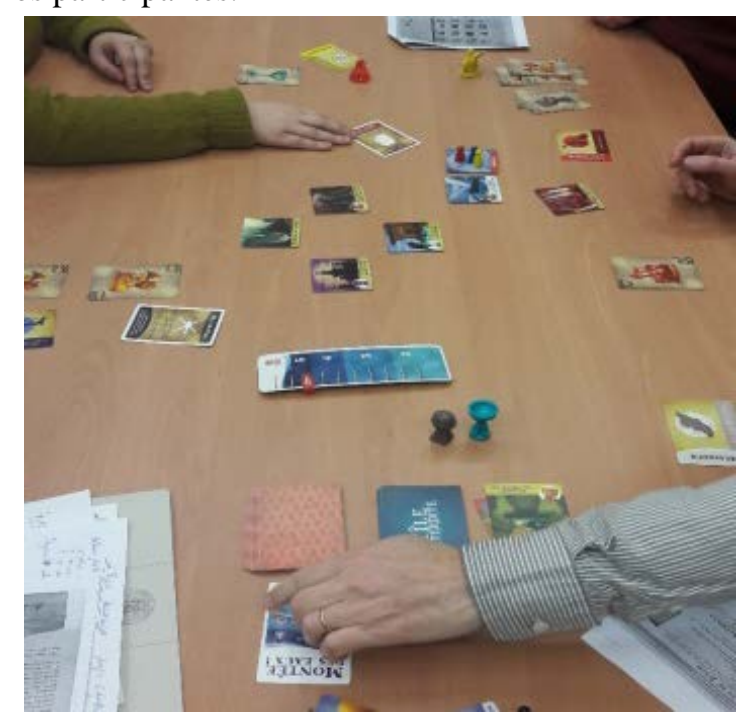

Fig. 3. Juego La isla prohibida

El testeo de los juegos, por parte del equipo docente que desarrolla el PIME, se realizó entre los meses de diciembre de 2017 y febrero de 2018, y los resultados obtenidos se abordan en las próximas líneas.

\section{Resultados}

Las pruebas realizadas a los juegos señalados arrojan el siguiente diagnóstico:

- $\quad$ El juego Honshú potencia el individualismo y la competitividad, pedagógicamente no aporta el aprendizaje de trabajo en equipo y cooperación, sin embargo, desde el punto de vista de la didáctica en ordenación de territorio y urbanismo, facilita el aprendizaje en el ámbito de la estructuración del territorio, incorpor conceptos como el de zona de valor ecológico y recursos naturales, y trabaja otros como los de de compacidad, continuidad y sostenibilidad medioambiental. Además, el sistema de puntuación permite la aproximación a la evaluación multicriterio. No obstante, es un modelo de juego sin roles ni jerarquías, lo que nos aleja de la realidad socio-territorial.

- $\quad$ El juego que más incide en las competencias transversales de trabajo en equipo, es $L a$ isla prohibida. La incorporación de esta competencia en el aprendizaje del estudiante,

(c)) BY-NC-ND 2018, Universitat Politècnica de València

Congreso IN-RED (2018) 
entronca con los principios de cooperación y colaboración, esenciales para alcanzar o aproximarnos al concepto y el hacer hacia la sostenibilidad. Además, el juego introduce el riesgo territorial como punto base, y estructura a los participantes en roles que tienen que les obliga a coordinarse en sus acciones, pero por el contrario, anula la competitividad. Con todo lo descrito también supone un modelo de juego que se aleja de la realidad socio-territorial.

- El juego Catán presenta un modelo de sociedad capitalista librecambista: control y dominio de territorios, recursos, comunicaciones y personas. Por tanto sí que refleja, en este caso, la realidad socio-territorial, afectada por jerarquías. Desde el punto de vista estructural nos enseña que la formación de poblados y ciudades está condicionada a elementos de localización y conectividad, y las vías de comunicación sólo puede bordear recursos territoriales, nunca atravesarlos. Además, permite incorporar al escenario límites al crecimiento: el recurso es agotable (petróleo) y su uso puede provocar efectos negativos (contaminación, catástrofes medio ambientales, etc.). Desde el punto de vista socio-económico la dinámica del juego sólo permite negociaciones y transacciones comerciales, no existe la cooperación, generando espacios (jugadores) segregados tanto físicamente como en su acceso a los recursos. Presenta por tanto, un concepto extremo de explotación del territorio y enseña lo contrario a la sostenibilidad.

De los resultados aquí recogidos, ninguno de estos juegos comerciales y analizados permite su inclusión completa y directa en didácticas de ordenación del territorio, puesto que no incluyen principios de colaboración y de competitividad, que nos lleven hacia la sostenibilidad. Se sitúan dentro de extremos opuestos: o cooperación u oposición; la combinación de ambos principios no se produce. Sin embargo, cada uno de los juegos testeados proporcionan elementos y técnicas de juego interesantes que adecuadamente combinados permitiría definir un modelo propio de juego que mejorase el aprendizaje profundo, y por tanto los resultados de aprendizaje, de los alumnos de Urbanismo y Ordenación del Territorio, fin ultimo del PIME que aquí se presenta.

\section{Conclusiones}

El aprendizaje lúdico como estrategia didáctica en materias de Ordenación del Territorio y Urbanismo, a través del diseño de un juego de mesa, es el reto que se propone alcanzar el equipo docente que compone el PIME El territorio en juego, de modo que se genere una metodología activa, construyendo un entorno de enseñanza-aprendizaje que estimule al alumnado a crear su propio conocimiento y experiencia, esencial para que subsuma principios y conceptos básicos de la complejidad territorial.

Uno de los primeros pasos, esenciales para alcanzar este reto, es testear los juegos comerciales que se acercan en su temática a la materia docente, de modo que podamos probarlos para extraer aprendizajes que podamos volcar en un nuevo diseño.

Utilizando una metodología basada en técnicas implicativas y reflexivas, se testean, por el profesorado participante en el PIME, tres juegos comercializados con el fin de investigar

(cc) EY-Nc-No 2018, Universitat Politècnica de València

Congreso In-Red (2018) 
cómo llevar la complejidad territorial al aula de forma tangible y lúdica. Las pruebas realizadas en juegos comercializados, nos sitúan ante situaciones que se posicionan en los extremos: absolutamente cooperativo-absolutamente competitivo, siendo la colaboración un instrumento comercial y la sostenibilidad un principio escasamente contemplado o anulado.

La creación y diseño de un juego didáctico para la ordenación del territorio deberá acercarse, desde distintos niveles, a la complejidad socio-territorial, donde cooperación y oposición pueden ocurrir casi simultáneamente entre los diferentes agentes, donde el trabajo en equipo pueda ser fundamental en la definición de estrategias y donde la concepción de la sostenibilidad en la creación, construcción y desarrollo del territorio esté presente en la simbología y códigos del juego.

\section{Referencias}

CHACÓN, P. (2008) . “ El Juego Didáctico como estrategia de enseñanza y aprendizaje. ¿Cómo crearlo en el aula?” Nueva Aula Abierta, $\mathrm{n}^{\circ}$ 16. <http://www.ehistoria.cl/cursosudla/13-EDU413/lecturas/06\%20\%20El\%20Juego\%20Didactico\%20Como\%20Estrategia\%20de\%20Ense\%C3\%B1anza\% 20y\%20Aprendizaje.pdf $>$ [Consulta: 22 de marzo 2018]

DELORS, J. (1996). La educación encierra un tesoro 2. Informe a la UNESCO de la Comisión Internacional para la Educación del siglo XXI. Santillana, Ediciones UNESCO $<$ http://www.unesco.org/education/pdf/DELORS_S.PDF > [Consulta: 20 de marzo 2017].

GAETE-QUEZADA, R.A. (2011). "El juego de roles como estrategia de evaluación de aprendizajes universitarios”. Educación y Educadores, Vol. 14, n $\mathrm{n}^{\circ}$ 2, pp. 289-307 $<$ http://educacionyeducadores.unisabana.edu.co/index.php/eye/article/view/1923/2512> $>$ [Consulta: 23 de marzo 2017]

GONZÁLEZ GONZÁLEZ, C.S. (2014). "Estrategias para trabajar la creatividad en la Educación Superior: pensamiento de diseño, aprendizaje basado en juegos y en proyectos". RED. Revista de Educación a Distancia, $\mathrm{n}^{\circ} \quad 40, \quad$ pp. $1-15$ $<$ http://revistas.um.es/red/article/view/234291/180001> [Consulta: 04 de abril 2017]

MONTAÑÉS SERANO, M. (2009). “Técnicas al servicio del proceso investigador”. En Montañés Serrano, M. Metodología y práctica participativa. Teoría y práctica de una estrategia de investigación participativa. Barcelon: Editorial UOC, pp. 127-170.

TAYLOR, J. (1983). Guía sobre simulación y juegos para la educación ambiental. UNESCO-PNUMA <http://unesdoc.unesco.org/images/0005/000569/056905so.pdf > [Consulta: 03 de abril 2017].

SOSA ESPINOSA, A.; PEREZ ALONSO, Y. (2014). "Role playing: Una buena herramienta para aprender”. En Martí Selva, ML.; Calafat Marzal, MC. (2014). I Jornadas de Investigación de la Facultad de ADE. Editorial Universitat Politècnica de València. Disponible en $<$ https://riunet.upv.es/handle/10251/43966 $>$ [Consulta: 5 de abril de 2017].

(cc) EY-NC-ND 2018, Universitat Politècnica de València

Congreso IN-RED (2018) 
Asenet Sosa Espinosa, Eric Gielen, José Sergio Palencia Jiménez, Yaiza Pérez Alonso, María Salomé Moreno Navarro, Rafael Ramón Temes Córdovez, Jose Luis Miralles Garcia, Maria Mercedes

Trenor Galindo

RIPOLL, O. (2006). “El juego como herramienta educativa”. Educación social: Revista de intervención socioeducativa. Núm. 33, pp. 11-27

$<$ http://www.raco.cat/index.php/EducacioSocial/article/viewFile/165533/373756>

[Consulta: 2 de febrero de 2018].

(c) EY-NC-ND 2018, Universitat Politècnica de València

Congreso In-Red (2018) 